\title{
Defining adult asthma endotypes by clinical features and patterns of volatile organic compounds in exhaled air
}

\author{
Norbert Meyer ${ }^{1,2^{*}}$, Jan W Dallinga ${ }^{3^{*}}$, Sarah Janine Nuss ${ }^{1}$, Edwin JC Moonen $^{3}$, Joep JBN van Berkel ${ }^{3}$, Cezmi Akdis $^{4}$, \\ Frederik Jan van Schooten ${ }^{3}$ and Günter Menz ${ }^{1}$
}

\begin{abstract}
Background: Several classifications of adult asthma patients using cluster analyses based on clinical and demographic information has resulted in clinical phenotypic clusters that do not address molecular mechanisms. Volatile organic compounds (VOC) in exhaled air are released during inflammation in response to oxidative stress as a result of activated leukocytes. VOC profiles in exhaled air could distinguish between asthma patients and healthy subjects. In this study, we aimed to classify new asthma endotypes by combining inflammatory mechanisms investigated by VOC profiles in exhaled air and clinical information of asthma patients.
\end{abstract}

Methods: Breath samples were analyzed for VOC profiles by gas chromatography-mass spectrometry from asthma patients ( $n=195)$ and healthy controls $(n=40)$. A total of 945 determined compounds were subjected to discriminant analysis to find those that could discriminate healthy from asthmatic subjects. 2-step cluster analysis based on clinical information and VOCs in exhaled air were used to form asthma endotypes.

Results: We identified 16 VOCs, which could distinguish between healthy and asthma subjects with a sensitivity of $100 \%$ and a specificity of $91.1 \%$. Cluster analysis based on VOCs in exhaled air and the clinical parameters FEV1, FEV1 change after 3 weeks of hospitalization, allergic sensitization, Junipers symptoms score and asthma medications resulted in the formation of 7 different asthma endotype clusters. We identified asthma clusters with different VOC profiles but similar clinical characteristics and endotypes with similar VOC profiles, but distinct clinical characteristics.

Conclusion: This study demonstrates that both, clinical presentation of asthma and inflammatory mechanisms in the airways should be considered for classification of asthma subtypes.

Keywords: Asthma, Endotypes, Phenotype, Volatile organic compounds, Exhaled air, Cluster

\section{Background}

Asthma is a chronic inflammatory disorder of the airways, which causes typical clinical symptoms like wheezing, breathlessness and chest tightness associated with a reversible obstruction of the airways. The heterogeneity of the clinical dimensions of asthma lead to the definition of clinical asthma phenotypes, classified by steroid response, obesity, fixed airway obstruction or trigger factors like

\footnotetext{
* Correspondence: norbert.meyer@insel.ch; j.dallinga@maastrichtuniversity.nl ${ }^{1}$ High Altitude Clinic (Hochgebirgsklinik) Davos, Davos-Wolfgang, Switzerland ${ }^{3}$ Department of Toxicology, Nutrition and Toxicology Research Institute Maastricht (Nutrim), Maastricht University Medical Center, Maastricht, The Netherlands

Full list of author information is available at the end of the article
}

allergens, air pollution, cigarette smoke, aspirin and exercise [1]. Using cluster analyses based on clinical and demographic information, adult asthma patients could be classified into 5 different subgroups [2], and children with severe asthma could be divided into 4 phenotypic clusters [3]. It is assumed that the clinical asthma phenotypes are associated with different inflammatory pathways or molecular mechanisms and on the other hand, similar molecular mechanisms may be present in different asthma phenotypes. In this context, the term "asthma endotypes" was introduced. Endotypes describe disease entities, which are functionally defined by distinct clinical features and pathologically by a molecular mechanism and treatment 
response $[4,5]$. It is proposed that the classification of patients into endotypes is important for establishing effective preventive measures and determining appropriate individualized treatments [1]. For quantification of molecular mechanisms non-invasive methods, reflecting the local airway inflammation, are important approaches.

Analysis of volatile organic compounds (VOC) in exhaled breath is a useful and non-invasive method for the assessment of airway inflammation [6]. For the collection of exhaled breath, three to four normal exhalations are necessary, which could be easily performed in children or in severe asthma patients with very low expiratory volumes [7]. VOCs are present in ambient air as well as result from metabolic processes in many cells and microorganisms. They are mainly released during inflammation from cells in response to oxidative stress as a result of activated leukocytes, which produce reactive oxygen species (ROS) [8]. ROS degrade cell membranes by lipid peroxidation and convert polyunsaturated fatty acids to VOCs, which are exhaled in the breath. In the human breath hundreds of different VOCs can be detected, which are divided into hydrocarbons, oxygen, or sulfur containing compounds and nitrogen containing substances [9]. The relative composition and concentrations of VOCs may alter in the presence of diseases and characteristic VOC patterns in lung diseases like lung cancer [10], cystic fibrosis [11], chronic obstructive pulmonary disease [12] and asthma [7] have been demonstrated. VOC profiles in exhaled air may distinguish between asthma patients and patients with chronic obstructive pulmonary disease [13]. In asthma patients, VOC patterns have been identified, which could specifically distinguish between allergic and non-allergic asthma [7] suggesting that different asthma phenotypes may be characterized by different VOC profiles in exhaled air. In addition, a recent study demonstrate that VOC profiles in exhaled air could classify clinically relevant disease phenotypes based on sputum inflammatory profile [14] and that VOC profiles might predict steroid responsiveness in mild/moderate asthma patients [15].

In this study, we measured VOCs in exhaled air from asthma patients and used the VOC profiles together with clinical characteristics for the formation of asthma clusters. We wanted to test the hypothesis that asthma clusters with similar clinical characteristics but different VOC patterns in exhaled air or clusters with similar VOC patterns but different clinical characteristics exist.

\section{Methods}

\section{Study design and asthma patients}

Adult patients had a physician diagnosis of asthma according to GINA guidelines (Global Initiative for Asthma, www.ginasthma.org). All patients included in the study were treated for at least 3 weeks at the high altitude clinic Davos-Wolfgang, which is located at $1600 \mathrm{~m}$ above sea level in the Swiss Alpine. The clinical and examination parameters were assessed when the patient arrived and after 3 weeks. All patients were recruited during a period of 8 months. The asthma patients were admitted to the high altitude clinic to optimize their disease. Therefore, mild, moderate and severe asthma patients were included. To exclude chronic obstructive pulmonary disease, asthma was defined by a reversibility in forced expiratory volume in $1 \mathrm{~s}$ (FEV1) of at least $12 \%$ predicted after inhalation of a short acting $\beta 2$-agonist. Asthma patients with an acute respiratory infection were excluded. To classify these patients as atopic or non-atopic, we evaluated their medical history, and skin prick tests were performed with animal dander, food allergens, pollens, fungi and latex. The NIOX system from Aerocrine was used for FENO (exhaled nitric oxide) measurements, according to the manufacturer's instructions. Blood eosinophils and eosininophilic cationic protein (ECP) were analysed in the laboratory of the high altitude clinic. The multidisciplinary treatment at high altitude, consisting of personalised treatment plans with physiotherapy and education, aimed to achieve full asthma control with the lowest possible dose of asthma medication. For the assessment of the level of asthma control the six-item Asthma Control Questionnaire (Junipers symptom score) was used [16]. Responses to each item are rated on a six-point scale and subsequently the mean was calculated, which ranged between 0 (totally controlled) and 6 (severely uncontrolled). Informed consent was obtained from all asthma patients. The main characteristics of the asthma patients are shown in Table 1. The study was approved by the local ethical committees of Graubünden and of Zürich. The data was stored in a database and analyzed by SPSS 18 (SPSS Schweiz AG, Zürich, Switzerland) and Graphpad Prism 4 (Graphprism Software, La Jolla, California). Healthy controls living in Davos were selected without any diseases and no allergies in their history during the same period of the study.

\section{Collection of exhaled air}

Exhaled air was collected between 07.00 and $07.30 \mathrm{~h}$ from all asthma patients and healthy subjects before breakfast. The subjects were asked to exhale their breath in a resistance-free plastic bag (Tedlar bag, SKC Ltd, Dorset, UK). Three to four exhalations were sufficient to fill the $5 \mathrm{l}$ bag. No special provisions for the mode of exhalation, nor special directions concerning the diet of the subjects were given. The samples of both patients and controls were collected in the same room, to prevent the occurrence of a background bias. Therefore, and because of the data analysis methods used (i.e. discriminant analysis), no background samples were collected. Within $1 \mathrm{~h}$ of collection, the bag was emptied under pressure over a stainless-steel two-bed sorption tube, 
Table 1 Characteristics of asthma patients

\begin{tabular}{ll}
\hline Allergic asthma & $\mathbf{7 7 . 9 \%}$ \\
\hline Age (years) & $49.8 \pm 15.2$ \\
Age of onset (years) & $23.5 \pm 17.0$ \\
Gender (male) & $44.6 \%$ \\
Smoker & $4.6 \%$ \\
Long-acting $\beta 2$ agonist & $92.8 \%$ \\
Short-acting $\beta 2$ agonist (puffs per day) & $1.8 \pm 3.2$ \\
Systemic steroids & $26.1 \%$ \\
Inhaled steroids & $94.9 \%$ \\
Theophylline & $21.0 \%$ \\
Junipers symptoms score & $2.2 \pm 1.2$ \\
ATS & $30.2 \%$ \\
Serum ECP ( $\mu$ g/l) & $22.9 \pm 18.6$ \\
Blood eosinophils (\%) & $5.6 \pm 3.8$ \\
FEV1 (\% of predicted normal value) & $83.2 \pm 23.5$ \\
\hline Percentages of male asthma patients, asthma patients who are active smokers, \\
asthma patients fulfilling ATS (American Thoracic Society) criterias for severe \\
asthma, asthma patients with an allergic asthma and asthma patients with \\
theophylline, inhaled long acting $\beta 2$ agonists, inhaled steroids or systemic \\
steroids as treatment are shown. In addition, year of onset, age, serum ECP \\
(eosinophilic cationic protein), blood eosinophils, FEV1 values are shown. The \\
units are indicated in the brackets.
\end{tabular}

filled with carbograph 1TD/Carbopack X (Markes International, Llantrisant, Wales, UK) and stored at room temperature until analysis.

\section{VOC's analysis}

For the analyses, the desorption tubes were placed inside the thermal desorption unit (Marks Unity desorption unit, Marks International Limited, Llantrisant, Wales, UK) and quickly heated to $270^{\circ} \mathrm{C}$ in order to release all VOCs and transport the released VOCs onto the GCcapillary. The used desorption unit was highly suitable for repeated, quantitative and reproducible measurements. Ten percent of the sample was injected into the $\mathrm{GC}$, the remaining $90 \%$ transported to another adsorption tube for storage and any later re-analysis. Just before the sample enters the GC, the sample is trapped by a cold trap at $5^{\circ} \mathrm{C}$ in order to focus and concentrate the sample. The VOCs were separated by capillary gas chromatography (column: RTX-5 ms, $30 \mathrm{~m} \times 0.25 \mathrm{~mm}$ 5\% diphenyl, 95\% dimethylsiloxane, film thickness $1 \mu \mathrm{m}$, Thermo Electron Trace GC Ultra, Thermo Electron Corporation, Waltham, USA). The temperature of the gas chromatograph was programmed as follows: $40^{\circ} \mathrm{C}$ during $5 \mathrm{~min}$, then raised with $10^{\circ} \mathrm{C} / \mathrm{min}$ until a final maximum temperature of $270^{\circ} \mathrm{C}$. This temperature was maintained for $5 \mathrm{~min}$. Time-of-flight mass spectrometry (TOF-MS) (Thermo Electron Tempus Plus time-of-flight mass spectrometer, Thermo Electron Corporation, Waltham, USA) was used to detect and identify components available in the samples. Electron ionization mode was set at $70 \mathrm{eV}$ and the mass range $m / z 35-350$ was measured at a scan rate of 0.2 s. All chromatograms were preprocessed [6] and combined into a final data matrix, which was used for the discriminant analyses.

\section{Cluster formation and statistical analyses}

Variables for cluster analyses were selected on the basis of their considered contribution for the characterization of asthma endotypes. Categorical variables were standardized to 0 or 1 and continuous variables were standardized to a continuous scale between 0 and 1 . For cluster analysis of categorical and continuous variables 2 -step cluster analysis approach using the program IBM SPSS Statistics 18.0 (IBM Corporation, New York, United States) was performed. To avoid the formation of very small clusters, the maximum cluster number was limited to 8 . Mean values of examination results or clinical features were calculated and are shown as mean \pm standard deviation (SD). All statistical analyses were performed using SPSS version 18.0 (IBM) and Graph Prism version 4.0 (GraphPad Software Inc, San Diego, CA).

\section{Results}

\section{Identification of VOCs implicated in the pathogenesis of} asthma

To investigate, which VOCs are implicated during asthmatic airway inflammation, VOCs in exhaled air were compared between asthma patients and healthy controls. A total of 945 VOCs were found. To select the most relevant VOCs, a classification model was constructed based on 16 VOCs. With this model it was possible to distinguish between asthma patients and healthy controls with a sensitivity of $100 \%$ and specificity of $91.1 \%$ if 16 components were used. 5 components were still sufficient to achieve a sensitivity of $100 \%$ and specificity of $85.3 \%$ (Figure 1A). This model with 16 VOCs was able to classify healthy and asthma subjects $98.7 \%$ correctly. The model with 5 VOCs still classified the subjects $98.0 \%$ correctly (Figure 1B). The chemical structures of the VOCs were partly identified and are shown (Additional file 1: Table S1).

\section{Formation of asthma endotypes}

For the formation of asthma endotype clusters by clinical features, asthma medications and VOCs in exhaled air unsupervised hierarchical two-step cluster analysis was used. The clinical features included forced expiratory volume in one-second (FEV1), its ratio to the inspiratory vital capacity (IVC), FEV1 improvement after 3 weeks in the high altitude clinic and Junipers asthma symptom score assessed at admittance. The therapy features included the fraction of patients using systemic steroids, inhaled steroids or long $\beta 2$ agonists and the frequency of 

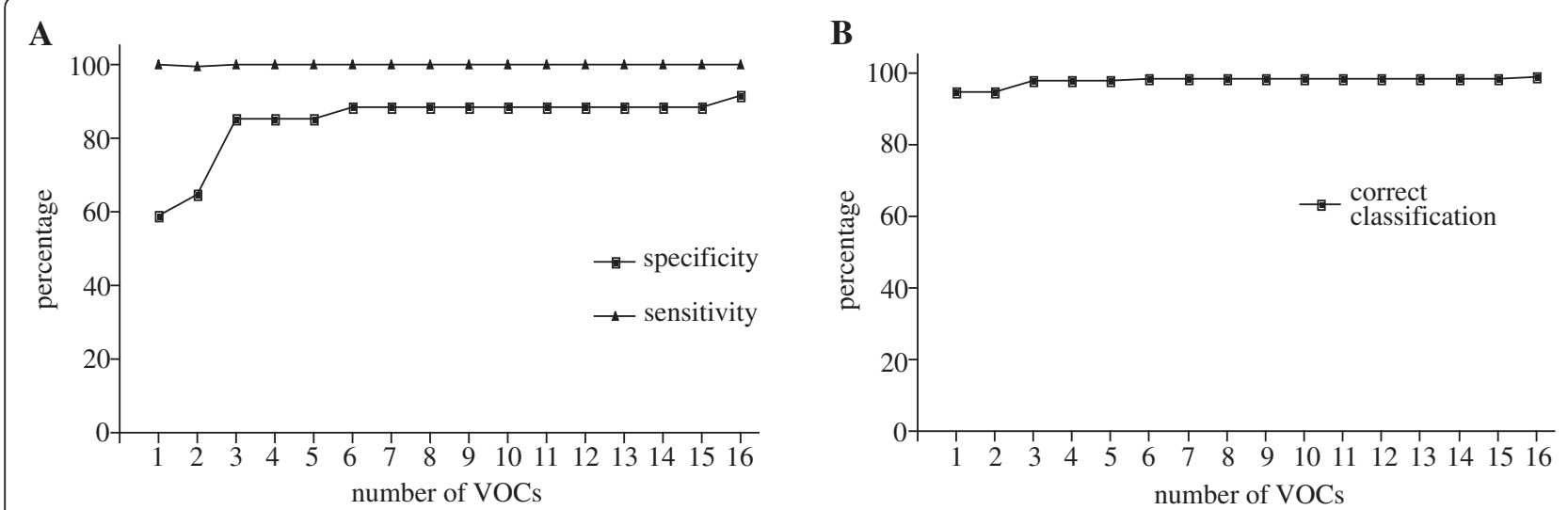

Figure 1 Course of sensitivity and specificity as a function of the number of VOCs involved in the discriminant analyses. The sensitivity and specificity to discriminate between asthma and healthy subjects depends on the number of VOCs (A). The correct classification of asthma and healthy subjects as a function of the VOCs is presented (B).

short $\beta 2$ agonist usage per day. To have an equal proportion between clinical parameters, asthma therapy features and VOCs in exhaled air for cluster analysis, we selected 4 VOCs for cluster analysis, which were detectable in asthma but not in healthy subjects (Figure 2). In detail, VOCs 141, 424, 470, 478 were selected (Figure 2A).

Unsupervised hierarchical two-step cluster analysis of clinical features, asthma medications and 4 VOCs divided the asthma patients into 7 different clusters (Table 2). The two smallest clusters included 14 patients (cluster 4 and 7) and the largest cluster 40 patients (cluster 2). Asthma patients in cluster 6 and 7 have a non-allergic asthma. The asthma patients of cluster 7 have a more severe disease compared to the asthma patients in cluster 6. In detail, asthma patients in cluster 7 have all systemic steroids as medications, a lower FEV1 and higher asthma symptoms scores compared to asthma patients in cluster 6 (Table 2).

In addition, 5 subgroups of patients with an allergic asthma were identified (cluster 1-5). Asthma patients in cluster 3 showed the most severe disease of allergic asthma with a high Junipers symptoms score, a low FEV1 but patients in cluster 3 did not use systemic steroids for asthma therapy. Asthma patients in clusters 1 and 2 had similar clinical features, including good FEV1 values, low Junipers symptoms scores, and no systemic steroid usage as asthma medications. Asthma patients in cluster 4 have a mild allergic asthma with similar clinical characteristics like asthma patients in cluster 1 and 2 but receive different asthma medications. Cluster 5 identifies asthma patients with a moderate allergic asthma having systemic steroids as asthma medication (for more details see Table 2).

More clinical and demographic parameters including age of onset, gender, body mass index (BMI), smoking habits, American thorax society (ATS) criteria for severe asthma, exhaled nitric oxide, serum eosinophilic cationic protein (ECP), circulating eosinophils, IgE serum levels, and usage of theophylline, which were not used for the cluster analysis, are shown in Table 3.

\section{Characterization of VOC profiles in asthma endotypes}

The concentrations of VOCs in the asthma clusters are shown in Table 2. To investigate if the VOCs were different or similar in the identified asthma clusters, we analysed if the VOCs could discriminate between them. Linear discriminant analysis demonstrates that $42.8 \%$ of all asthma patient clusters and healthy subjects could be correctly classified by VOCs in the exhaled air (Figure 3A). However, the correct classification was different between the clusters indicating that there exist clusters with similar VOC profiles and clusters with different VOC profiles. Subsequent analyses demonstrate that cluster 1 and 2 have similar clinical features (Table 2) but different VOC patterns in exhaled air (Figure 3B). Profiles of VOCs can discriminate between cluster 1, 2 and healthy subjects with an high accuracy of $95.3 \%$ (Figure 3B). In contrast, the correct classification of asthma patients belonging to cluster 6 and 7 and healthy donors was only $81.8 \%$ and there was much overlap of the VOC patterns between these clusters indicating that these asthma clusters have similar VOC patterns in exhaled air (Figure 3C). Interestingly, asthma patients belonging to cluster 6 and 7 have distinct clinical and treatment characteristics (see Table 2). Similar results were found for cluster 3 and 4 (Figure 3D), which are clinical different but have a quite related profile of VOCs in exhaled air as indicated by the relatively low accuracy of $82.9 \%$ to distinguish between the asthma patients clusters and healthy donors.

In addition, a second possibility of cluster analysis was performed. In this data set, the following additional parameters were included: age of onset, blood eosinophils, body mass index (BMI), serum IgE levels, exhaled nitric 


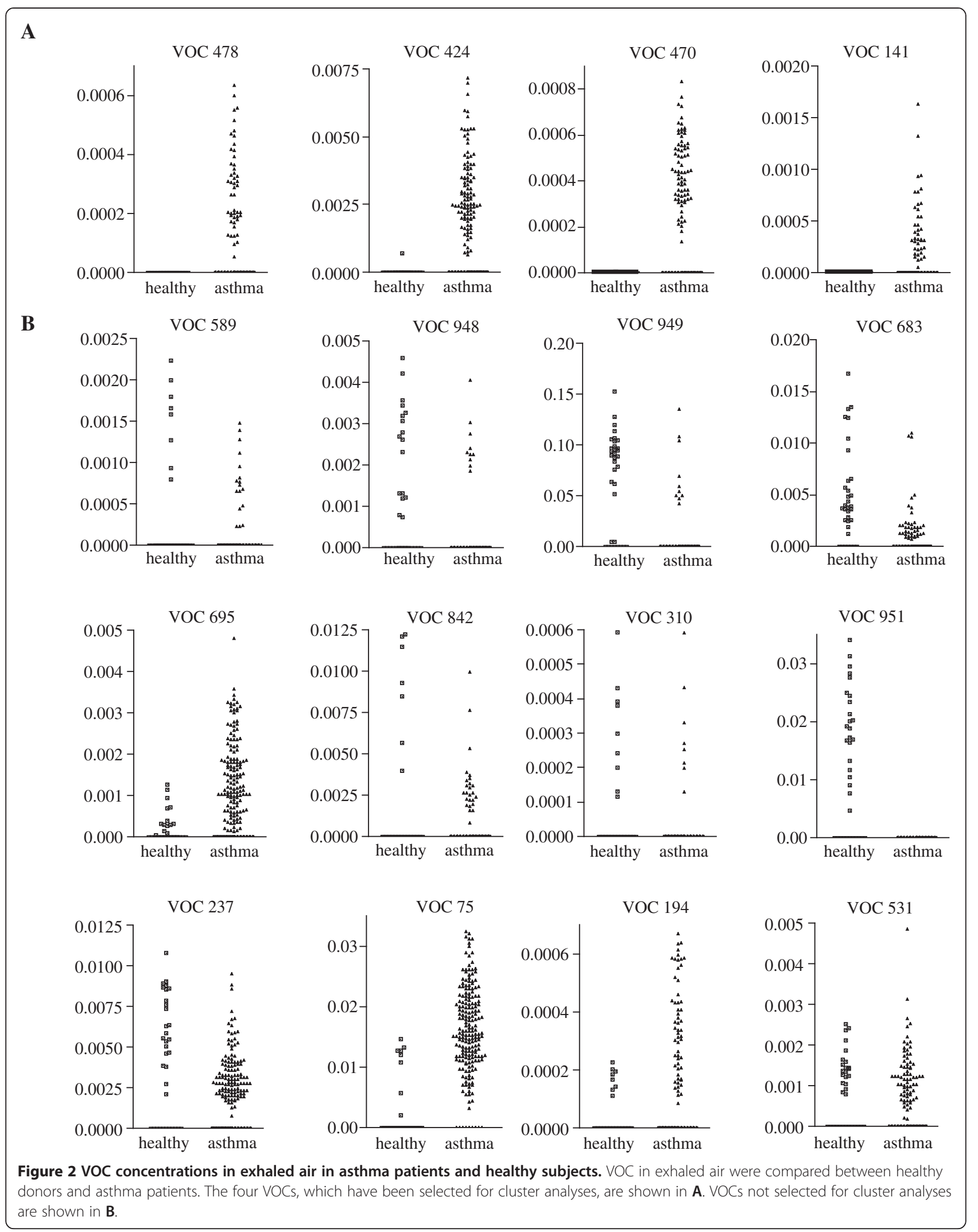


Table 2 Values for cluster formation

\begin{tabular}{llllllll}
\hline Parameter & Cluster 1 & Cluster 2 & Cluster 3 & Cluster 4 & Cluster 5 & Cluster 6 & Cluster 7 \\
\hline $\mathrm{n}$ & 33 & 40 & 28 & 14 & 37 & 29 & 14 \\
Allergies & $100 \%$ & $100 \%$ & $100 \%$ & $100 \%$ & $100 \%$ & $0 \%$ & $0 \%$ \\
FEV1 & $99.7 \pm 15.8$ & $96.7 \pm 16.3$ & $58.7 \pm 14.5$ & $105.0 \pm 17.2$ & $73.1 \pm 18.0$ & $84.4 \pm 18.1$ & $58.3 \pm 22.5$ \\
FEV1/IVC & $0.99 \pm 0.09$ & $0.98 \pm 0.1$ & $0.77 \pm 0.15$ & $1.02 \pm 0.05$ & $0.91 \pm 0.18$ & $0.92 \pm 0.17$ & $0.71 \pm 0.21$ \\
FEV1 improvement & $-4.9 \pm 11.8$ & $1.3 \pm 7.6$ & $8.6 \pm 14.6$ & $-0.4 \pm 6.9$ & $2.1 \pm 14.0$ & $1.0 \pm 11.3$ & $14.8 \pm 21.3$ \\
Junipere & $1.8 \pm 1.3$ & $1.6 \pm 1.0$ & $3.2 \pm 0.8$ & $0.9 \pm 0.6$ & $2.8 \pm 1.0$ & $1.7 \pm 0.9$ & $2.9 \pm 1.1$ \\
Systemic steroids & $0 \%$ & $0 \%$ & $0 \%$ & $7.1 \%$ & $97.3 \%$ & $0 \%$ & $100 \%$ \\
Inhaled steroids & $100 \%$ & $100 \%$ & $100 \%$ & $42.9 \%$ & $100 \%$ & $96.6 \%$ & $92.9 \%$ \\
Long $\beta 2$ mimetics & $100 \%$ & $100 \%$ & $100 \%$ & $0 \%$ & $100 \%$ & $100 \%$ & $100 \%$ \\
Short $\beta 2$ mimetics & $0.52 \pm 0.94$ & $0.68 \pm 1.25$ & $3.93 \pm 4.74$ & $0.57 \pm 1.40$ & $3.05 \pm 3.54$ & $1.10 \pm 1.76$ & $3.79 \pm 5.32$ \\
VOC_141 & $0.015 \pm 0.069$ & $0.191 \pm 0.246$ & $0.042 \pm 0.119$ & $0.020 \pm 0.076$ & $0.163 \pm 0.381$ & $0.056 \pm 0.165$ & $0.034 \pm 0.112$ \\
VOC_470 & $0.033 \pm 0.090$ & $0.360 \pm 0.253$ & $0.238 \pm 0.241$ & $0.126 \pm 0.228$ & $0.062 \pm 0.143$ & $0.214 \pm 0.268$ & $0.174 \pm 0.251$ \\
VOC_478 & 0 & $0.152 \pm 0.175$ & $0.041 \pm 0.112$ & $0.012 \pm 0.046$ & $0.053 \pm 0.128$ & $0.104 \pm 0.179$ & $0.147 \pm 0.195$ \\
VOC_424 & $0.436 \pm 0.988$ & $3.228 \pm 1.751$ & $1.070 \pm 1.654$ & $1.938 \pm 1.717$ & $0.933 \pm 1.502$ & $1.921 \pm 2.031$ & $1.816 \pm 1.828$ \\
\hline
\end{tabular}

Percentages of patients with allergies, systemic steroids, inhaled steroids and inhaled long acting $\beta 2$ agonists for asthma treatment are shown. FEV1 (forced expiratory volume in 1 second) values are shown in the percentage of the predicted normal values for FEV1. For the Junipers symptoms score and short $\beta 2$ agonists the mean values \pm SD are indicated. Unit for inhalation of short $\beta 2$ agonists is puffs per day.

oxide (FeNO) and asthma control. Because unsupervised cluster resulted in the formation of only 3 clusters, a 2 step cluster analysis of this data set using a user-defined cluster number of 7 was performed. We have detected two clusters with a non-allergic asthma and 5 clusters with an allergic asthma (see Additional file 1: Table S2). Again, clusters with a high clinical similarity but distinct profiles of exhaled VOCs (e.g. cluster 2 and 3, Additional file 1: Figure S1B) and clusters with similar VOCs patterns in exhaled air but distinct clinical and treatment characteristics (e.g. clusters 2 and 4, Additional file 1: Figure S1C) are present. Interestingly, cluster 2 and 3 have not only distinct VOCs profiles, they also differ in total IgE levels and blood eosinophils. In contrast, patients in cluster 2 and 4 are clinical different and have similar VOC profiles and IgE/blood eosinophil levels.

\section{Discussion}

The current study identifies VOCs in exhaled air, which could distinguish between asthma patients and healthy subjects with a sensitivity of $100 \%$ and specificity of $91 \%$. The combination of clinical asthma features, asthma therapy and exhaled VOCs classified asthma patients into 7 endotype clusters. We identified asthma clusters

Table 3 Additional clinical characteristics of asthma clusters

\begin{tabular}{|c|c|c|c|c|c|c|c|c|}
\hline Parameter & Cluster 1 & Cluster 2 & Cluster 3 & Cluster 4 & Cluster 5 & Cluster 6 & Cluster 7 & $\mathbf{n}$ \\
\hline Age of onset (years) & $20.7 \pm 16.3$ & $19.5 \pm 15.4$ & $19.0 \pm 17.9$ & $26.5 \pm 20.5$ & $24.1 \pm 17.1$ & $28.9 \pm 14.8$ & $34.3 \pm 16.2$ & 195 \\
\hline Gender (male) & $36.4 \%$ & $52.5 \%$ & $60.1 \%$ & $35.7 \%$ & $37.8 \%$ & $51.7 \%$ & $21.4 \%$ & 195 \\
\hline Age & $41.6 \pm 15.9$ & $46.4 \pm 15.9$ & $56.6 \pm 12.1$ & $47.3 \pm 14.6$ & $49.5 \pm 13.4$ & $54.4 \pm 12.4$ & $58.9 \pm 16.4$ & 195 \\
\hline $\mathrm{BMI}$ & $25.0 \pm 3.6$ & $25.5 \pm 3.1$ & $26.0 \pm 4.3$ & $25.9 \pm 4.2$ & $25.3 \pm 4.7$ & $24.7 \pm 3.8$ & $24.7 \pm 5.0$ & 195 \\
\hline Tobacco exposure (py) & $4.2 \pm 6.0$ & $3.3 \pm 7.7$ & $6.0 \pm 8.0$ & $1.8 \pm 4.1$ & $4.2 \pm 7.1$ & $5.0 \pm 8.6$ & $2.0 \pm 3.8$ & 195 \\
\hline Smoker & $6.1 \%$ & $2.5 \%$ & $7.1 \%$ & $7.1 \%$ & $5.4 \%$ & $3.5 \%$ & $0 \%$ & 195 \\
\hline ATS & $9.1 \%$ & $12.5 \%$ & $35.7 \%$ & $0 \%$ & $75.7 \%$ & $6.9 \%$ & $78.6 \%$ & 195 \\
\hline Exhaled NO (ppb) & $36 \pm 29$ & $32 \pm 18$ & $34 \pm 30$ & $27 \pm 27$ & $43 \pm 36$ & $28 \pm 18$ & $41 \pm 26$ & 195 \\
\hline $\mathrm{ECP}$ & $27.0 \pm 27.0$ & $21.7 \pm 13.8$ & $26.2 \pm 20.8$ & $11.9 \pm 7.2$ & $22.3 \pm 16.1$ & $18.9 \pm 15.6$ & $31.1 \pm 19.8$ & 194 \\
\hline Eosinophils & $6.5 \pm 4.5$ & $5.6 \pm 3.1$ & $6.3 \pm 3.6$ & $4.8 \pm 5.0$ & $4.8 \pm 3.1$ & $4.8 \pm 3.8$ & $7.2 \pm 4.2$ & 195 \\
\hline $\lg \mathrm{E}$ & $1383 \pm 4731$ & $524 \pm 883$ & $648 \pm 1007$ & $304 \pm 340$ & $392 \pm 877$ & $80 \pm 103$ & $140 \pm 262$ & 194 \\
\hline Theophyllin & $12.1 \%$ & $12.5 \%$ & $32.1 \%$ & $0 \%$ & $32.4 \%$ & $10.3 \%$ & $57.1 \%$ & 195 \\
\hline
\end{tabular}

Percentages of male asthma patients, asthma patients who are active smokers, asthma patients fulfilling ATS (American Thoracic Society) criterias for severe asthma and asthma patients with theophylline treatment are shown. In addition, the BMI (body mass index), year of onset, age, exhaled NO (nitric oxide), serum ECP (eosinophilic cationic protein), blood eosinophils, lgE serum levels are shown as mean values \pm SD. The units are indicated in the brackets. Lifetime tobacco exposure is indicated in pack years (py). 
A

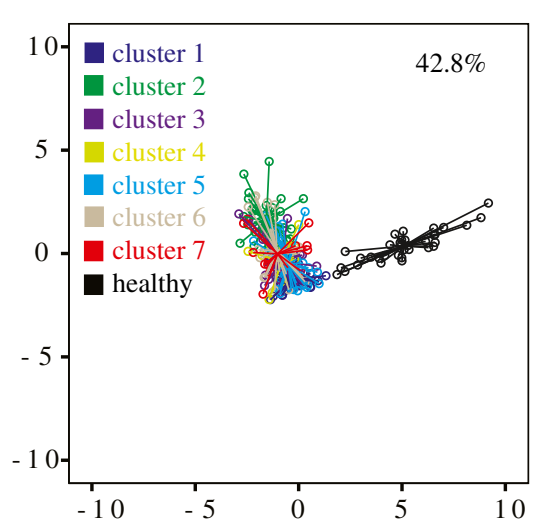

C

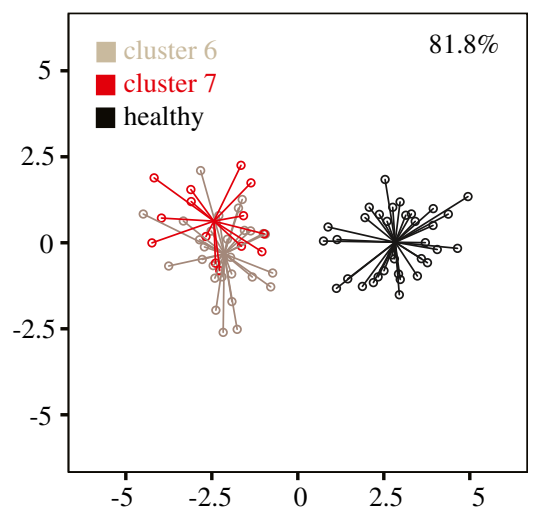

B

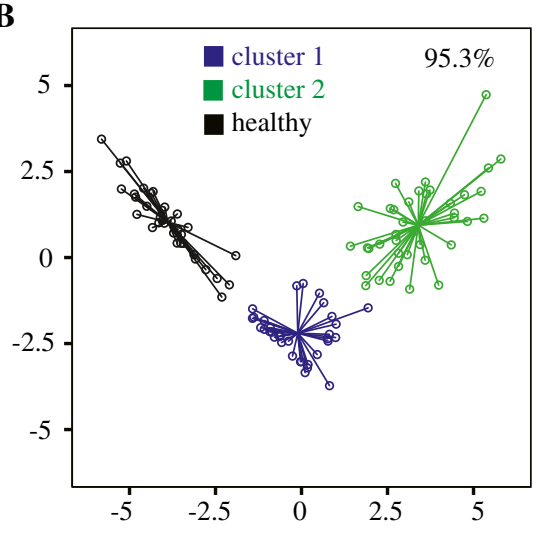

D

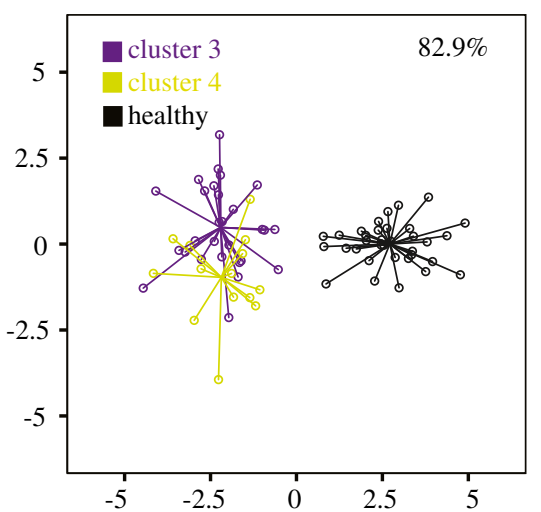

Figure 3 Discriminant analyses for asthma clusters. Discrimination analyses between all asthma clusters and healthy patients (A) and between indicated asthma subgroups (B, C, D) are shown. The percentage indicates the correct classification. Clusters shown in B have similar phenotype characteristics whereas clusters shown in $\mathbf{C}$ and $\mathbf{D}$ have different phenotype characteristics (see Table 2).

with different VOC profiles but similar clinical characteristics and clusters with similar VOC profiles, but distinct clinical characteristics.

The activation of different cellular and molecular pathways by different environmental factors suggests that asthma phenotypes are associated with certain inflammatory pathways in the lungs. Asthma endotypes classify asthma patients according to clinical features, treatment response and underlying molecular mechanisms and therefore address molecular mechanism [4]. Accordingly, we used 13 parameters of clinical features, asthma treatment features and VOCs in exhaled air for cluster analysis and classified all asthma patients into 7 different clusters. Recent phenotype studies of severe adult asthma patients or asthmatic children identified 4-5 asthma groups by cluster analysis without addressing molecular mechanisms or including exhaled VOCs for cluster analyses [2,3]. The identification of asthma patient clusters with similar clinical and treatment characteristics but different VOC profiles in the exhaled air indicates that asthma phenotypes might be further divided into subgroups by their exhaled VOC profiles. This finding supports the hypothesis that one asthma phenotype might be characterized by several inflammatory mechanisms [5]. The identification of molecular mechanisms specific for asthma endotypes and diagnostic biomarkers that can discriminate between distinct disease endotypes will be necessary for the development of new asthma therapies [17]. We also detected asthma clusters with similar VOC patterns in the exhaled air but different clinical features suggesting that these VOCs profiles may be specific for airway inflammation, which is found in many asthma phenotypes.

In this study VOCs in exhaled air were analysed for the assessment of airway inflammation because VOCs mostly derive from biological processes such as lipid peroxidation. Pathologic processes have the potential to influence VOCs either by producing new volatile substances or by the metabolic consumption of VOC substrates that are normally present. Volatile products formed during lipid peroxidation include ethane, pentane, hexanal, octanal, nonanal, propanol and butanol $[18,19]$. Already in the early 80 s, pentane and ethane levels in exhaled air were described as markers for in vivo peroxidation [8]. Ethane was described to be elevated in the breath of patients with 
chronic obstructive pulmonary disease (COPD) and asthma $[20,21]$. However, measuring a single compound in exhaled air might not be sufficient to monitor complex and heterogeneous diseases. In addition, some VOCs in exhaled air are not only increased during airway inflammation. For example, pentane appears to be relatively non-specific VOC for airway inflammation because its levels are also raised in other inflammatory conditions such as inflammatory bowel disease [22] or rheumatoid arthritis [23]. Exploring the total amount of VOCs is expected to generate more adequate information about the complexity of processes underlying the pathophysiology of respiratory diseases [24]. In our study the most important 16 VOCs could distinguish with a high sensitivity and specificity between healthy and asthmatic subjects indicating that the VOCs are useful for non-invasive exploration of various biochemical pathways activated in asthma.

Certain inflammatory processes may reflect the involvement of different inflammatory sputum cells. Thus, it has been demonstrated that VOC profiles in exhaled air could classify clinically relevant disease phenotypes based on sputum inflammatory profiles by predicting both neutrophilic and eosinophilic sputum cell phenotypes of asthma patients [14]. However, a variety of interactions among distinct populations of stromal cells, epithelial cells and leukocytes contribute to inflammation in the asthmatic lung [25] and may not be assessed by characterization of cells derived from sputa. Analysis of gene expression in asthmatic biopsies might be a useful technique to understand the inflammatory processes implicated in airway inflammation [26,27]. However, obtaining lung biopsies is invasive and cannot be assessed in all asthma patients. In contrast, breath analysis could detect VOC profiles in a non-invasive and straightforward way.

Although this study does not identify VOCs, which indicate specific inflammatory mechanisms in the airways, we demonstrate that VOCs patterns are an attractive approach for the classification of asthma patients as they might reflect inflammatory processes in the airways and could be collected easily in a non-invasive way. If we include biological relevant features of blood analyses like blood eosinophils and IgE serum levels into the cluster analyses, we detect clusters having similar clinical asthma features (see Additional file 1: Table S2) but differ in IgE levels, blood eosinophils and VOCs suggesting that certain VOCs profiles might indicate IgEand eosinophil-mediated inflammation. The identification of VOCs as markers for certain inflammatory pathways in asthma patients (e.g. Th2- or Th-17 cell mediated inflammation) might be the basis to guide the patients individual therapy.

Therefore the identification of VOCs, which are specifically related to the expression of certain genes and proteins is one important step in the future, which could be helpful for the identification of discrete pathogenic pathways specific for asthma endotypes.

\section{Additional file}

Additional file 1: Table S1. Identified chemical VOC structures. Table S2. Second possibility of cluster analysis. Figure S1. Discriminant analyses for asthma clusters.

\section{Competing interests}

The authors declare that they have no competing interests.

\section{Authors' contributions}

NM collected the data, carried out the cluster analyses, designed the experiments and drafted the manuscript. JD analysed the data of volatile organic compounds and drafted the manuscript. SN collected the patients data, performed the collection of volatile organic compounds and contributed to the data analyses. EM performed the GC-analyses. CA supported us with the study design and experimental setup. FS contributed to the study design and to the VOC analyses. GM was responsible for the study design, manuscript draft and collection of clinical data. All authors read and approved the final manuscript.

\section{Author details}

${ }^{1}$ High Altitude Clinic (Hochgebirgsklinik) Davos, Davos-Wolfgang, Switzerland. ${ }^{2}$ Clinic for Rheumatology, Immunology and Allergology, Divison of Allergology, University Hospital of Bern, Bern, Switzerland. ${ }^{3}$ Department of Toxicology, Nutrition and Toxicology Research Institute Maastricht (Nutrim), Maastricht University Medical Center, Maastricht, The Netherlands. ${ }^{4}$ Swiss Institute of Allergy and Asthma Research (SIAF), University of Zurich, Christine-Kühne Center for Allergy Research and Education (CK-CARE), Davos, Switzerland.

Received: 13 May 2014 Accepted: 21 October 2014

Published online: 28 November 2014

\section{References}

1. Wenzel SE: Asthma: defining of the persistent adult phenotypes. Lancet 2006, 368:804-813.

2. Moore WC, Moore WC, Meyers DA, Wenzel SE, Teague WG, Li H, Li X, D'Agostino R Jr, Castro M, Curran-Everett D, Fitzpatrick AM, Gaston B, Jarjour NN, Sorkness R, Calhoun WJ, Chung KF, Comhair SA, Dweik RA, Israel E, Peters SP, Busse WW, Erzurum SC, Bleecker ER: Identification of asthma phenotypes using cluster analysis in the Severe Asthma Research Program. Am J Respir Crit Care Med 2009, 181:315-323.

3. Fitzpatrick AM, Teague WG, Meyers DA, Peters SP, Li X, Li H, Wenzel SE, Aujla S, Castro M, Bacharier LB, Gaston BM, Bleecker ER, Moore WC: Heterogeneity of severe asthma in childhood: confirmation by cluster analysis of children in the National Institutes of Health/National Heart, Lung, and Blood Institute Severe Asthma Research Program. J Allergy Clin Immunol 2011, 127:382-389. e1.

4. Anderson GP: Endotyping asthma: new insights into key pathogenic mechanisms in a complex, heterogeneous disease. Lancet 2008, 372:1107-1119.

5. Lotvall J, Akdis CA, Bacharier LB, Bjermer L, Casale TB, Custovic A, Lemanske RF Jr, Wardlaw AJ, Wenzel SE, Greenberger PA: Asthma endotypes: a new approach to classification of disease entities within the asthma syndrome. J Allergy Clin Immunol 2011, 127(2):355-360.

6. Van Berkel JJ, Dallinga JW, Moller GM, Godschalk RW, Moonen E, Wouters EF, Van Schooten FJ: Development of accurate classification method based on the analysis of volatile organic compounds from human exhaled air. J Chromatogr B Analyt Technol Biomed Life Sci 2008, 861:101-107.

7. Dallinga JW, Robroeks CM, van Berkel JJ, Moonen EJ, Godschalk RW, Jobsis Q, Dompeling E, Wouters EF, van Schooten FJ: Volatile organic compounds in exhaled breath as a diagnostic tool for asthma in children. Clin Exp Allergy 2009, 40:68-76. 
8. Pauling $L$, Robinson $A B$, Teranishi $R$, Cary P: Quantitative analysis of urine vapor and breath by gas-liquid partition chromatography. Proc Natl Acad Sci U S A 1971, 68:2374-2376.

9. Miekisch W, Schubert JK, Noeldge-Schomburg GF: Diagnostic potential of breath analysis-focus on volatile organic compounds. Clin Chim Acta 2004, 347:25-39.

10. Phillips M, Gleeson K, Hughes JM, Greenberg J, Cataneo RN, Baker L, McVay WP: Volatile organic compounds in breath as markers of lung cancer: a cross-sectional study. Lancet 1999, 353:1930-1933.

11. Robroeks CM, van Berkel JJ, Dallinga JW, Jobsis Q, Zimmermann LJ, Hendriks HJ, Wouters MF, van der Grinten CP, van de Kant KD, van Schooten FJ, Dompeling E: Metabolomics of volatile organic compounds in cystic fibrosis patients and controls. Pediatr Res 2010, 68:75-80.

12. Van Berkel JJ, Dallinga JW, Moller GM, Godschalk RW, Moonen EJ, Wouters EF, Van Schooten FJ: A profile of volatile organic compounds in breath discriminates COPD patients from controls. Respir Med 2009, 104:557-563.

13. Fens N, Zwinderman AH, van der Schee MP, de Nijs SB, Dijkers E, Roldaan AC, Cheung D, Bel EH, Sterk PJ: Exhaled breath profiling enables discrimination of chronic obstructive pulmonary disease and asthma. Am J Respir Crit Care Med 2009, 180:1076-1082.

14. Ibrahim B, Basanta M, Cadden P, Singh D, Douce D, Woodcock A, Fowler SJ: Non-invasive phenotyping using exhaled volatile organic compounds in asthma. Thorax 2011, 66:804-809.

15. van der Schee MP, Palmay R, Cowan JO, Taylor DR: Predicting steroid responsiveness in patients with asthma using exhaled breath profiling. Clin Exp Allergy 2013, 43:1217-1225.

16. Juniper EF, O'Byrne PM, Guyatt GH, Ferrie PJ, King DR: Development and validation of a questionnaire to measure asthma control. Eur Respir J 1999, 14:902-907.

17. Akdis CA: Therapies for allergic inflammation: refining strategies to induce tolerance. Nat Med 2012, 18:736-749.

18. Dillard CJ, Tappel AL: Lipid peroxidation products in biological tissues. Free Radic Biol Med 1989, 7:193-196.

19. Orhan $\mathrm{H}$ : Analyses of representative biomarkers of exposure and effect by chromatographic, mass spectrometric, and nuclear magnetic resonance techniques: method development and application in life sciences. J Sep Sci 2007, 30:149-174.

20. Paredi P, Kharitonov SA, Barnes PJ: Elevation of exhaled ethane concentration in asthma. Am J Respir Crit Care Med 2000, 162:1450-1454.

21. Paredi P, Kharitonov SA, Leak D, Ward S, Cramer D, Barnes PJ: Exhaled ethane, a marker of lipid peroxidation, is elevated in chronic obstructive pulmonary disease. Am J Respir Crit Care Med 2000, 162:369-373.

22. Kokoszka J, Nelson RL, Swedler WI, Skosey J, Abcarian H: Determination of inflammatory bowel disease activity by breath pentane analysis. Dis Colon Rectum 1993, 36:597-601.

23. Humad S, Zarling E, Clapper M, Skosey JL: Breath pentane excretion as a marker of disease activity in rheumatoid arthritis. Free Radic Res Commun 1988, 5:101-106

24. Boots AW, van Berkel JJ, Dallinga JW, Smolinska A, Wouters EF, van Schooten FJ: The versatile use of exhaled volatile organic compounds in human health and disease. J Breath Res 2012, 6:027108.

25. Lloyd CM, Hessel EM: Functions of $T$ cells in asthma: more than just $T(H) 2$ cells. Nat Rev Immunol 2010, 10(12):838-848.

26. Dougherty RH, Sidhu SS, Raman K, Solon M, Solberg OD, Caughey GH, Woodruff PG, Fahy JV: Accumulation of intraepithelial mast cells with a unique protease phenotype in $\mathrm{T}(\mathrm{H}) 2$-high asthma. J Allergy Clin Immunol 2010, 125:1046-1053. e8.

27. Bisgaard H, Pipper CB, Bonnelykke K: Endotyping early childhood asthma by quantitative symptom assessment. J Allergy Clin Immunol 2011, 127:1155-1164. e2.

doi:10.1186/s12931-014-0136-8

Cite this article as: Meyer et al:: Defining adult asthma endotypes by clinical features and patterns of volatile organic compounds in exhaled air. Respiratory Research 2014 15:136.

\section{Submit your next manuscript to BioMed Central and take full advantage of:}

- Convenient online submission

- Thorough peer review

- No space constraints or color figure charges

- Immediate publication on acceptance

- Inclusion in PubMed, CAS, Scopus and Google Scholar

- Research which is freely available for redistribution

Submit your manuscript at www.biomedcentral.com/submit 